PROCEEDINGS OF THE

AMERICAN MATHEMATICAL SOCIETY

Volume 131, Number 11, Pages 3353-3364

S 0002-9939(03)07070-9

Article electronically published on May 5, 2003

\title{
VANISHING THEOREMS, BOUNDEDNESS AND HYPERBOLICITY OVER HIGHER-DIMENSIONAL BASES
}

\author{
SÁNDOR J. KOVÁCS \\ (Communicated by Michael Stillman)
}

\begin{abstract}
A vanishing theorem is proved for families over higher dimensional bases and applied to generalize some Shafarevich type statements to that setting.
\end{abstract}

Let $C$ be a smooth projective curve of genus $g$ over an algebraically closed field of characteristic $0, \Delta \subset C$ a finite subset and $q>1$ a positive integer. Let $\delta=\# \Delta$. Shafarevich conjectured that the set of non-isotrivial families of smooth projective curves of genus $q$ over $C \backslash \Delta$ is finite; furthermore if $2 g-2+\delta \leq 0$, then there are no such families. This was confirmed by Parshin68] for the case $\Delta=\emptyset$ and by Arakelov71] in general; cf. Beauville81]. For a more detailed discussion see Bedulev-Viehweg00.

Recently there has been a flurry of new results regarding higher-dimensional generalizations of this problem: Migliorini95, Kovács96, Kovács97b, [Bedulev-Viehweg00, [Kovács00a], [Viehweg-Zuo01a], and [Kovács00b]. All of these articles study generalizations when the base of the family is a curve.

The main goal of the present article is to extend (some of) these results to higherdimensional bases, i.e., replacing the fixed curve, $C$, with a fixed smooth projective variety, $S$, of arbitrary dimension.

An extension like that may be useful for several reasons. A consequence of the results for curves can be phrased in the following way: An isotrivial family of curves of genus at least 2 over $\mathbb{P}^{1}$ must have at least 3 singular fibers. In other words the corresponding moduli space has a certain hyperbolic property. This, of course, implies similar statements for families over a base that is covered by rational curves, e.g., $\mathbb{P}^{k}$, but not statements as strong as one would expect. Consider a family that is smooth over $\mathbb{P}^{k} \backslash \Delta$ where $\Delta$ is a suitable effective divisor. Then the above statement implies that $\operatorname{deg} \Delta>2$. However, if the hyperbolic behavior holds over higher-dimensional bases as well, then one expects that $\operatorname{deg} \Delta>k+1$ or equivalently that $\omega_{\mathbb{P}^{k}}(\Delta)$ be ample. In particular, this would also say that certain families defined over a plane curve cannot be extended to a family over $\mathbb{P}^{2}$.

One can go even further and conjecture that if the variation of the family $f$ : $X \rightarrow S$ in moduli is maximal, then $\omega_{S}(\Delta)$ is big. For a more precise formulation of

Received by the editors April 4, 2001 and, in revised form, June 10, 2002.

2000 Mathematics Subject Classification. Primary 14J10.

This work was supported in part by NSF Grants DMS-0196072, DMS-0092165, and a Sloan Research Fellowship. 
these conjectures see Conjecture 5.2.1 These are special cases of Viehweg's more general conjectures [Viehweg00 6.3]. The last part of Shafarevich's conjecture is a special case of this, and so are the other known generalizations of that.

The proofs of the known cases center around various Kodaira type vanishing theorems. One expects that the first step toward a proof of this conjecture would be a new vanishing theorem.

The main result of this article is a vanishing theorem. It can be considered a relative version of the logarithmic Kodaira-Akizuki-Nakano vanishing theorem. For the precise statement see Theorem 2.5. The proof of this theorem occupies $\S \S 1$ and 2. $\S 3$ is devoted to some positivity results for the push-forward of powers of the relative dualizing sheaf. The proofs here closely follow ideas of Kollár and Viehweg. As an application of the results in $\S \S 2$ and 3, a boundedness result is proved for families over higher-dimensional bases in $\S 4$. The interesting point here is more the technique than the result itself.

Another application is presented in $\S 5$. A couple of theorems are proved for the degeneracy locus of certain families of varieties of general type over a base that is one of the following: An abelian variety, $\mathbb{P}^{k}$ for $k \leq 3$, or a quadric hypersurface of dimension at most three. Theorem 5.1 .1 is new; it treats a slightly more general case than what was previously known Kovács97a, 0.1]. Theorems [5.2.2 and 5.2.4 are unfortunately not as strong as expected. In particular, they also follow from the more general Viehweg00, 2.6]. Furthermore, in Theorems [5.2.2 and [5.2.4 the restrictions on the dimension of the base and fibers respectively should not be necessary. In fact, they are not present in the vanishing theorem 2.5. The problem is in the application part. The assumptions of the vanishing theorem hold trivially in the cases presented here, and there is no obvious reason why they would not apply in a more general situation. To resolve this issue will require more work, but one may hope that this method will actually lead to a more general statement along the lines of Conjecture 5.2.1, Note that since the writing of this article, Viehweg-Zuo01b. achieved stronger results in this direction with different methods.

Definitions and notation. Throughout the article the groundfield will always be $\mathbb{C}$, the field of complex numbers. The dimension of the empty set is $-\infty$.

Let $f: X \rightarrow S$ be a morphism of schemes. Then $X_{s}$ denotes the fiber of $f$ over the point $s \in S$ and $f_{s}$ denotes the restriction of $f$ to $X_{s}$. Similarly, $X_{\text {gen }}$ denotes the generic fiber of $f$. For a coherent sheaf $\mathcal{F}, \mathcal{F}^{*}$ denotes its dual, $\mathcal{H o m}\left(\mathcal{F}, \mathcal{O}_{X}\right)$.

Let $f: X \rightarrow S$ be a surjective morphism between smooth projective varieties. $\operatorname{Var}(f)$ denotes the number of effective parameters of the birational equivalence classes of the fibers. For the rigorous definition of $\operatorname{Var}(f)$ the reader should consult Kollár87 or [Viehweg83a. $f$ will be called isotrivial if $\operatorname{Var}(f)=0$.

A line bundle $\mathcal{L}$ on $X$ is called big if $X$ is proper and the global sections of $\mathcal{L}^{m}$ define a birational map for some $m>0$, and it is called nef if $\operatorname{deg}\left(\left.\mathcal{L}\right|_{C}\right) \geq 0$ for every proper curve $C \subset X$. In particular, ample implies nef and big. $\mathcal{L}$ will be called $l$-big if $X$ is proper and the global sections of $\mathcal{L}^{m}$ define a rational map, $\phi: X \rightarrow Y$, for some $m>0$ such that $\operatorname{dim} X-\operatorname{dim} \phi(X) \leq l$. Obviously 0-big is the same as big. Let $U$ be an open subset of $X . \mathcal{L}$ is called semi-ample with respect to $U$ if some positive power of $\mathcal{L}$ is generated by global sections over $U$, i.e., the natural map $\left.H^{0}\left(X, \mathcal{L}^{b}\right) \otimes \mathcal{O}_{U} \rightarrow \mathcal{L}^{b}\right|_{U}$ is surjective for some $b>0$. It is called semi-ample if it is semi-ample with respect to $X$. Similarly $\mathcal{L}$ is called l-ample with respect to $U$ if the global sections of some positive power of $\mathcal{L}$ define 
a rational map, $\phi: X \rightarrow Y$, such that $\left.\phi\right|_{U}$ is a morphism (i.e., defined everywhere on $U), \phi^{-1}(\phi(U))=U$, and $\operatorname{dim} \phi^{-1}(y) \leq l$ for all $y \in \phi(U)$. Let $X$ be a smooth projective variety and $U \subseteq X$ an open subset. Let $\mathcal{E}$ be a locally free sheaf on $X, \pi: P=\mathbb{P}(\mathcal{E}) \rightarrow X$ the projective bundle associated with $\mathcal{E}$ and $\mathcal{L}=\mathcal{O}_{\mathbb{P}(\mathcal{E})}(1)$ the corresponding tautological line bundle. $\mathcal{E}$ is called semi-positive or nef (resp. semi-ample with respect to $U$, resp. l-big, resp. l-ample with respect to $U$ ) if $\mathcal{L}$ on $P$ is nef (resp. semi-ample with respect to $\pi^{-1}(U)$, resp. $l$-big, resp. $l$-ample with respect to $\left.\pi^{-1}(U)\right)$.

\section{A VANISHING THEOREM FOR VECTOR BUNDLES}

An important ingredient of the main vanishing theorem in $\S 2$ is in turn a generalization of the Kodaira-Akizuki-Nakano vanishing theorem. This generalization is essentially a combination of the logarithmic version of Esnault and Viehweg and the vector bundle version of Le Potier. In fact the proof of this combined version does not require any new ideas.

1.1 Theorem ([Esnault-Viehweg92 6.7], LePotier75, Théorème 1]). Let $X$ be a smooth projective variety of dimension $n, D$ an effective normal crossing divisor and $\mathcal{E}$ a locally free sheaf of rank $r$ on $X$. Assume that $\mathcal{E}$ is semi-ample (with respect to $X)$, and $l$-big and $l+1$-ample with respect to $X \backslash D$ for some $l \in \mathbb{N}$. Then for $p+q \geq n+r+l$,

$$
H^{q}\left(X, \Omega_{X}^{p}(\log D)\left(-D_{r e d}\right) \otimes \mathcal{E}\right)=0 .
$$

Proof. The following lemma reduces the question to the line bundle case.

1.2 Le Potier's Lemma (cf. Shiffman-Sommese85, 5.16]). Let $\mathcal{F}$ be a coherent sheaf on $X, \pi: P=\mathbb{P}(\mathcal{E}) \rightarrow X$ the projective bundle associated to $\mathcal{E}$, and $\mathcal{L}=$ $\mathcal{O}_{\mathbb{P}(\mathcal{E})}(1)$ the corresponding tautological line bundle on $P$. Then for all $p, q \in \mathbb{N}$,

$$
H^{q}\left(X, \mathcal{F} \otimes \Omega_{X}^{p}(\log D) \otimes \mathcal{E}\right) \simeq H^{q}\left(P, \pi^{*} \mathcal{F} \otimes \Omega_{P}^{p}\left(\log \pi^{*} D\right) \otimes \mathcal{L}\right) .
$$

Proof. The proof of [Shiffman-Sommese85, 5.16] works word-for-word if one replaces $\Omega_{X}$ and $\Omega_{P}$ with $\Omega_{X}(\log D)$ and $\Omega_{P}\left(\log \pi^{*} D\right)$.

Now take $\mathcal{F}=\mathcal{O}_{X}$ and use Esnault-Viehweg92, 92, 4.11, 6.7] for $\mathcal{L}$ on $P$.

1.3 Remark. An alternative way is to use Esnault-Viehweg92, 2.3] and induction.

\section{VANISHING THEOREMS FOR FAMILIES}

2.1. Let $f: X \rightarrow S$ be a surjective morphism between smooth projective varieties with connected fibers, $\operatorname{dim} X=n$ and $\operatorname{dim} S=k$. Let $\Delta \subset S$ be an effective normal crossing divisor and assume that $D=f^{*} \Delta \subset X$ is also a normal crossing divisor. Assume that $(X, D) \rightarrow(S, \Delta)$ has the following properties: $X \backslash D \rightarrow S \backslash \Delta$ is smooth, and the sheaf $\Omega_{X / S}(\log D)$ defined by the short exact sequence

$$
0 \rightarrow f^{*} \Omega_{S}(\log \Delta) \rightarrow \Omega_{X}(\log D) \rightarrow \Omega_{X / S}(\log D) \rightarrow 0,
$$

is locally free. $\Omega_{X / S}^{q}(\log D)$ will denote $\Lambda^{q} \Omega_{X / S}(\log D)$.

2.2. Let $\mathcal{E}$ be a locally free sheaf of finite rank on $S$. Then there exists a filtration

$$
\mathcal{E}=\mathcal{F}^{0} \supset \mathcal{F}^{1} \supset \cdots \supset \mathcal{F}^{r}=0
$$


of $\mathcal{E}$ such that $\mathcal{F}^{i-1} / \mathcal{F}^{i}$ is a locally free sheaf of rank at most $k=\operatorname{dim} S$ for all $i=1, \ldots, r$. Choose such a filtration for each $\mathcal{E}$ on $S$ and let

$$
\mathfrak{F}(\mathcal{E})=\left\{\mathcal{F}^{i-1} / \mathcal{F}^{i} \mid \mathcal{F}^{i} \text { is the chosen filtration of } \mathcal{E}\right\} .
$$

These choices together will be called a choice of filtrations on $S$.

The following is a trivial, but important observation:

2.3 Lemma. Let $f: X \rightarrow S$ be as in subsection 2.1. If for some $i \in \mathbb{N}$ and $\mathcal{G}$ a coherent sheaf on $X, H^{i}\left(X, \mathcal{G} \otimes f^{*} \mathcal{E}\right) \neq 0$, then there exists an $\mathcal{E}^{\prime} \in \mathfrak{F}(\mathcal{E})$ such that $H^{i}\left(X, \mathcal{G} \otimes f^{*} \mathcal{E}^{\prime}\right) \neq 0$.

2.4 Definition. Let $f: X \rightarrow S$ be as in subsection 2.1 and let $\mathfrak{A}$ be a set of locally free sheaves of finite rank on $S$. Let $\mathfrak{T}^{0}(=A)=\mathfrak{A}$ and

$$
\mathfrak{T}(\mathfrak{A})=\mathfrak{T}^{1}(\mathfrak{A})=\bigcup \mathfrak{F}\left(\mathcal{E} \otimes \Omega_{S}^{q}(\log \Delta) \otimes \omega_{S}(\Delta)^{-1}\right),
$$

where the union is taken for all $\mathcal{E} \in \mathfrak{A}$ and for $q=0, \ldots, k$. Furthermore, for $r \geq 1$, let $\mathfrak{T}^{r}(\mathfrak{A})=\mathfrak{T}\left(\mathfrak{T}^{r-1}(\mathfrak{A})\right)$.

2.5 Theorem. Let $f: X \rightarrow S$ be as in subsection 2.1. Let $\mathfrak{A}$ be a set of locally free sheaves of rank at most $k$ on $S, \mathcal{L}$ a line bundle on $X$ such that for a suitable choice of filtrations on $S, \mathcal{L}\left(D_{\text {red }}\right) \otimes f^{*} \mathcal{E}^{\prime}$ is semi-ample (with respect to $X$ ), and big and 1 -ample with respect to $X \backslash D$ for all $\mathcal{E}^{\prime} \in \mathfrak{T}^{i}(\mathfrak{A}), i=0, \ldots, n-k$. Then for all $\mathcal{E} \in \mathfrak{A}$,

$$
H^{p}\left(X, \Omega_{X / S}^{q}(\log D) \otimes f^{*} \omega_{S}(\Delta) \otimes \mathcal{L} \otimes f^{*} \mathcal{E}\right)=0 \quad \text { for } p+q \geq n .
$$

Proof. The statement will be proved by induction using the following lemma.

2.6 Lemma. Let $p, q \in \mathbb{N}$ such that $p+q \geq n$ and assume that for every natural number $l>q$ and for every $\mathcal{E}^{\prime} \in \mathfrak{T}(\mathfrak{A})$,

$$
H^{p-1}\left(X, \Omega_{X / S}^{l}(\log D) \otimes f^{*} \omega_{S}(\Delta) \otimes \mathcal{L} \otimes f^{*} \mathcal{E}^{\prime}\right)=0 .
$$

Then for every $\mathcal{E} \in \mathfrak{A}, H^{p}\left(X, \Omega_{X / S}^{q}(\log D) \otimes f^{*} \omega_{S}(\Delta) \otimes \mathcal{L} \otimes f^{*} \mathcal{E}\right)=0$.

Proof. Let $\mathcal{E} \in \mathfrak{A}$. The standard exact sequence of $f$,

$$
0 \rightarrow f^{*} \Omega_{S}^{1}(\log \Delta) \rightarrow \Omega_{X}^{1}(\log D) \rightarrow \Omega_{X / S}^{1}(\log D) \rightarrow 0,
$$

yields a filtration on $\Omega_{X}^{q+k}(\log D) \otimes \mathcal{L} \otimes f^{*} \mathcal{E}$ which in turn induces a spectral sequence,

$$
\begin{gathered}
E_{1}^{r, s}=H^{r+s}\left(X, \Omega_{X / S}^{q+k-r}(\log D) \otimes f^{*} \Omega_{S}^{r}(\log \Delta) \otimes \mathcal{L} \otimes f^{*} \mathcal{E}\right), \\
E_{1}^{r, s} \Rightarrow H^{r+s}\left(X, \Omega_{X}^{q+k}(\log D) \otimes \mathcal{L} \otimes f^{*} \mathcal{E}\right)
\end{gathered}
$$

Since $p+q \geq n, H^{p}\left(X, \Omega_{X}^{q+k}(\log D) \otimes \mathcal{L} \otimes f^{*} \mathcal{E}\right)=0$ by Theorem 1.1. Hence $E_{\infty}^{r, p-r}=0$ for all $r$. In particular, $E_{\infty}^{k, p-k}=0$. Suppose now that

$$
E_{1}^{k, p-k}=H^{p}\left(X, \Omega_{X / S}^{q}(\log D) \otimes f^{*} \omega_{S}(\Delta) \otimes \mathcal{L} \otimes f^{*} \mathcal{E}\right) \neq 0 .
$$

Observe that $E_{w}^{u, v}=0$ for every $u>k$ and arbitrary $v, w$, so $E_{\infty}^{k, p-k}$ can be zero only if there exists a $t>0$ such that $E_{t}^{k-t, p-1-k+t} \neq 0$. Then $E_{1}^{k-t, p-1-k+t} \neq 0$ for the same $t$, so $l=q+t>q$ is such that

$$
\begin{gathered}
E_{1}^{k-t, p-1-k+t}=H^{p-1}\left(X, \Omega_{X / S}^{l}(\log D) \otimes f^{*} \Omega_{S}^{k-t}(\log \Delta) \otimes \mathcal{L} \otimes f^{*} \mathcal{E}\right) \neq 0, \text { i.e. } \\
H^{p-1}\left(X, \Omega_{X / S}^{l}(\log D) \otimes f^{*} \omega_{S}(\Delta) \otimes \mathcal{L} \otimes f^{*}\left(\mathcal{E} \otimes \Omega_{S}^{k-t}(\log \Delta) \otimes \omega_{S}(\Delta)^{-1}\right)\right) \neq 0 .
\end{gathered}
$$


Therefore $H^{p-1}\left(X, \Omega_{X / S}^{l}(\log D) \otimes f^{*} \omega_{S}(\Delta) \otimes \mathcal{L} \otimes f^{*} \mathcal{E}^{\prime}\right) \neq 0$, for some $\mathcal{E}^{\prime} \in \mathfrak{T}(\mathfrak{A})$ by Lemma 2.3 . This contradicts the hypotheses, hence the statement follows.

Proof of Theorem 2.5 continued. Let $\mathcal{E} \in \mathfrak{A}$ and suppose that

$$
H^{p}\left(X, \Omega_{X / S}^{q}(\log D) \otimes f^{*} \omega_{S}(\Delta) \otimes \mathcal{L} \otimes f^{*} \mathcal{E}\right) \neq 0
$$

for some $p, q$, such that $p+q \geq n$. Then by Lemma 2.6 there exist $\mathcal{E}_{1} \in \mathfrak{T}(\mathfrak{A})$ and $q_{1}>q$ such that

$$
H^{p-1}\left(X, \Omega_{X / S}^{q_{1}}(\log D) \otimes f^{*} \omega_{S}(\Delta) \otimes \mathcal{L} \otimes f^{*} \mathcal{E}_{1}\right) \neq 0
$$

Now $p-1+q_{1} \geq p+q \geq n$, so this step can be repeated. I.e., for $j=2, \ldots, n-k+1$, there exist $\mathcal{E}_{j} \in \mathfrak{T}^{j}(\mathfrak{A})$ and $q_{j}>q_{j-1}$ such that

$$
H^{p-j}\left(X, \Omega_{X / S}^{q_{j}}(\log D) \otimes f^{*} \omega_{S}(\Delta) \otimes \mathcal{L} \otimes f^{*} \mathcal{E}_{1}\right) \neq 0 .
$$

However, $q_{n-k+1}>n-k$, so $\Omega_{X / S}^{q_{n-k+1}}(\log D)=0$, a contradiction.

2.7 Remark. Note that the "extra" positivity assumption of Theorem 2.5 is asking positivity in a horizontal direction. In other words, if $\mathcal{L}$ is relatively ample, then the assumption asks that $\mathcal{L}$ be more positive than certain given sheaves coming from $S$. Therefore the applicability of the theorem does not depend as much on the family as it does on the base. In particular, if one studies families over a fixed base, with fixed degeneracy locus, then the assumptions provide a fixed threshold for the required positivity of $\mathcal{L}$ for all families over this base. This should be understood as a kind of boundedness statement for families over a fixed base with fixed degeneracy locus (or smooth families over $S \backslash \Delta$ ); cf. $\S 4$.

2.8 Corollary. Let $f: X \rightarrow S$ be as in subsection [2.1. Let $\mathcal{M}$ be a line bundle on $X$. Assume that $\mathcal{M}$ contains another line bundle $\mathcal{L}$ such that for a suitable choice of filtrations on $S, \mathcal{L}\left(D_{\text {red }}\right) \otimes f^{*} \mathcal{E}$ is semi-ample (with respect to $X$ ), and big and 1 -ample with respect to $X \backslash D$ for all $\mathcal{E} \in \mathfrak{T}^{i}\left(\mathcal{O}_{S}\right), i=0, \ldots, n-k$. Then

$$
H^{n}\left(X, f^{*} \omega_{S}(\Delta) \otimes \mathcal{M}\right)=0 .
$$

Proof. $H^{n}\left(X, f^{*} \omega_{S}(\Delta) \otimes \mathcal{L}\right)=0$ by Theorem 2.5. The cokernel of the embedding $\mathcal{L} \subseteq \mathcal{M}$ is supported in codimension 1 , so the map

$$
H^{n}\left(X, f^{*} \omega_{S}(\Delta) \otimes \mathcal{L}\right) \rightarrow H^{n}\left(X, f^{*} \omega_{S}(\Delta) \otimes \mathcal{M}\right)
$$

is surjective, while $H^{n}\left(X, f^{*} \omega_{S}(\Delta) \otimes \mathcal{L}\right)=0$.

2.9 Corollary. If in addition $f$ is smooth and $S$ is an abelian variety, then $H^{n}(X, \mathcal{K})=0$ for every line bundle $\mathcal{K}$ on $X$ that contains a big and 1 -ample line bundle.

\section{WEAK POSITIVITY}

3.1. Weakly positive sheaves. The following notation will be fixed for the rest of subsection 3.1. Let $S$ be a quasi-projective variety, and $U \subseteq S$ an open subset.

3.1.1 Definition (Viehweg83a $)$. Let $\mathcal{F}$ be a coherent torsion free sheaf on $S$ and $\iota: V \hookrightarrow S$ the maximal open subscheme such that $\left.\mathcal{F}\right|_{V}$ is locally free. We define the symmetric powers of $\mathcal{F}$ by $\operatorname{Sym}^{r}(\mathcal{F})=\iota_{*} \operatorname{Sym}^{r}\left(\left.\mathcal{F}\right|_{V}\right)$. 
Let $\mathcal{L}$ be an ample line bundle on $S$. Then $\mathcal{F}$ is called weakly positive over $U$ if $\left.\mathcal{F}\right|_{U}$ is locally free and for all $a>0$ there exists some $b>0$ such that $\operatorname{Sym}^{a b}(\mathcal{F}) \otimes \mathcal{L}^{b}$ is generated by global sections over $U$, i.e., the natural map

$$
\left.H^{0}\left(S, \operatorname{Sym}^{a b}(\mathcal{F}) \otimes \mathcal{L}^{b}\right) \otimes \mathcal{O}_{U} \rightarrow \operatorname{Sym}^{a b}(\mathcal{F}) \otimes \mathcal{L}^{b}\right|_{U}
$$

is surjective.

3.1.2 Lemma. Let $\mathcal{E}$ be a coherent sheaf on $S$. Assume that either $\mathcal{E}$ is an ample locally free sheaf or $\mathcal{E}=\mathcal{F} \otimes \mathcal{L}$, where $\mathcal{F}$ is a weakly positive sheaf over $U$ and $\mathcal{L}$ is an ample line bundle. Then there exists a $\lambda_{0}>0$ such that for all $\lambda$, divisible by $\lambda_{0}$, and for any $t_{1}, t_{2} \in U, t_{1} \neq t_{2}$, the natural map

$$
\rho_{\lambda}: H^{0}\left(S, \operatorname{Sym}^{\lambda}(\mathcal{E})\right) \rightarrow\left(\operatorname{Sym}^{\lambda}(\mathcal{E}) \otimes k\left(t_{1}\right)\right) \oplus\left(\operatorname{Sym}^{\lambda}(\mathcal{E}) \otimes k\left(t_{2}\right)\right)
$$

is surjective.

Proof. Let $\mathfrak{m}_{i}$ be the ideal sheaf of $\left\{t_{i}\right\}$ for $i=1,2$ and $\mathfrak{I}_{1,2}=\mathfrak{m}_{1} \cap \mathfrak{m}_{2}$ that of $\left\{t_{1}, t_{2}\right\}$.

Let $\mathcal{E}$ be an ample locally free sheaf. Then $H^{1}\left(S, \operatorname{Sym}^{\lambda}(\mathcal{E}) \otimes \mathfrak{I}_{1,2}\right)=0$ for $\lambda \gg 0$. In fact by semi-continuity there is a $\lambda_{0}$ such that this vanishing holds for all $\lambda \geq \lambda_{0}$ and for all pairs of points on $S$, so the statement follows.

Let $\mathcal{E}=\mathcal{F} \otimes \mathcal{L}$ as above. Since $\mathcal{L}$ is ample, there exists an $n>0$ such that $\mathcal{L}^{b n}$ is very ample for all $b>0$. Hence there exist sections $\sigma_{1}, \sigma_{2} \in H^{0}\left(S, \mathcal{L}^{b n}\right)$ such that $\sigma_{i} \in \mathfrak{m}_{i} \mathcal{L}^{b n}$, but $\sigma_{i} \notin \mathfrak{m}_{3-i} \mathcal{L}^{b n}$ for $i=1,2$. Now let $a=n+1$. Since $\mathcal{F}$ is weakly positive, there exists a $b_{0}>0$, such that $\operatorname{Sym}^{a b}(\mathcal{F}) \otimes \mathcal{L}^{b}$ is globally generated over $U$ for all $b \geq b_{0}$ Viehweg83b 3.2]. Then $\operatorname{Sym}^{a b}(\mathcal{E}) \simeq \operatorname{Sym}^{a b}(\mathcal{F}) \otimes \mathcal{L}^{b} \otimes \mathcal{L}^{b n}$, and using $\sigma_{i}$ one easily sees that $\operatorname{im} \rho_{a b}$ contains $\left(\operatorname{Sym}^{a b}(\mathcal{E}) \otimes k\left(t_{1}\right)\right) \oplus 0$ and $0 \oplus\left(\operatorname{Sym}^{a b}(\mathcal{E}) \otimes\right.$ $\left.k\left(t_{2}\right)\right)$. Therefore $\rho_{a b}$ is surjective, so the statement holds with $\lambda_{0}=a b_{0}$.

3.1.3 Lemma. Let $f: X \rightarrow S$ be a surjective morphism, $\mathcal{M}$ a line bundle on $X$, and $\mathcal{N}$ a line bundle on $S$. Assume that $\mathcal{M}_{t}=\left.\mathcal{M}\right|_{X_{t}}$ is generated by global sections for all $t \in U, f_{*} \mathcal{M}$ is locally free on $U$, and $f_{*} \mathcal{M}^{m}$ is reflexive on $S$ for all $m$ large and divisible enough. Assume further that for some $r>0$ there exists a subsheaf $\mathcal{E} \subseteq \operatorname{Sym}^{r}\left(f_{*}=M \otimes \mathcal{N}\right)$, such that $\operatorname{supp}\left(\operatorname{Sym}^{r}\left(f_{*} \mathcal{M} \otimes \mathcal{N}\right) / \mathcal{E}\right) \subseteq S \backslash U$, and either $\mathcal{E}$ is an ample locally free sheaf or $\mathcal{E}=\mathcal{F} \otimes \mathcal{L}$, where $\mathcal{F}$ is a weakly positive sheaf over $U$ and $\mathcal{L}$ is an ample line bundle on $S$. Then $\mathcal{M} \otimes f^{*} \mathcal{N}$ is big and semi-ample with respect to $X \backslash f^{-1} U$ and if $m$ is large and divisible enough, then the global sections of $\mathcal{M}^{m} \otimes f^{*} \mathcal{N}^{m}$ separate the fibers over $U$ and on $X_{t}$ induce the same map as do the sections of $\mathcal{M}_{t}^{m}$.

Proof. Since $\mathcal{M}_{t}$ is generated by global sections for $t \in U, f^{*} \operatorname{Sym}^{m}\left(f_{*} \mathcal{M}\right) \rightarrow \mathcal{M}^{m}$ is surjective on $X \backslash f^{-1} U$. In particular, $\alpha: f^{*} \operatorname{Sym}^{m}\left(f_{*} \mathcal{M}\right) \otimes\left(\mathcal{O}_{X_{t}} \oplus \mathcal{O}_{X_{s}}\right) \rightarrow$ $\mathcal{M}_{t}^{m} \oplus \mathcal{M}_{s}^{m}$ is surjective for $t, s \in U$. Now one has the following commutative diagram:

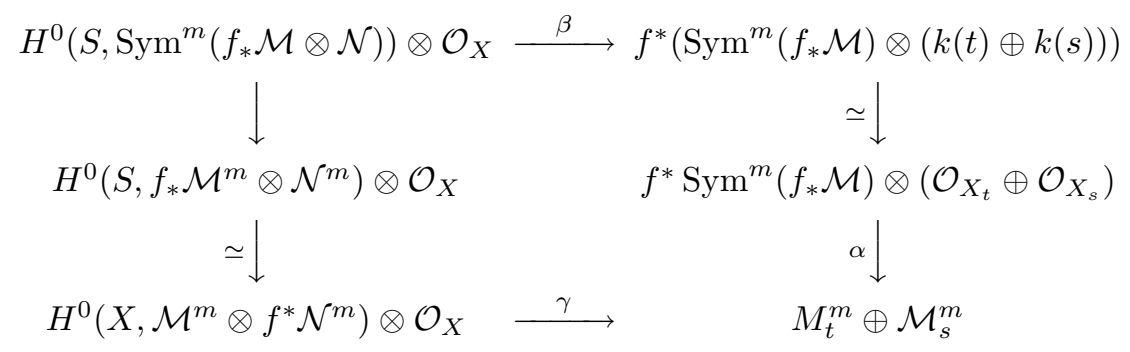


If $m$ is large and divisible enough, then $\beta$ is surjective by Lemma 3.1.2, hence $\alpha \circ \beta$ is surjective, and then so is $\gamma$. This proves the statement.

\subsubsection{Corollary.}

(3.1.4.1) If $\mathcal{M}_{t}$ is l-big for general $t \in S$, then $\mathcal{M} \otimes f^{*} \mathcal{N}$ is l-big.

(3.1.4.2) If $\mathcal{M}_{t}$ is l-ample for $t \in U$, then $\mathcal{M} \otimes f^{*} \mathcal{N}$ is l-ample with respect to $X \backslash f^{-1} U$.

\subsection{Weak positivity of push-forwards of powers of dualizing sheaves.}

3.2.1. Let $f: X \rightarrow S$ be a surjective morphism between smooth projective varieties with connected general fiber. Let $n=\operatorname{dim} X, k=\operatorname{dim} S$, and $\Delta \subset S$ denote the subset of $S$ such that $X \backslash f^{-1} \Delta \rightarrow S \backslash \Delta$ is smooth. Assume that $\omega_{X_{\text {gen }}}$ is nef and big, $\operatorname{Var}(f)=\operatorname{dim} S$, and if $m$ is large and divisible enough, then $f_{*} \omega_{X / S}^{m}$ is locally free on $S$.

3.2.2 Remark. The last technical assumption is likely avoidable. The rest of the assumptions imply that $f_{*} \omega_{X / S}^{m}$ is locally free on $S \backslash \Delta$. A possible way to try to avoid the extra assumption, at least for families of canonically polarized manifolds, is to assume that the minimal model program works in dimension $n$ and to use the results of [Karu00] on the existence of compactified moduli. Those allow one to find a birational model with the required property. Another possibility is to use the weak semistable reduction of [Abramovich-Karu00] as is done in Viehweg-Zuo01b and Viehweg-Zuo01c]. For details please consult the latter articles.

3.2.3 Lemma. Let $f: X \rightarrow S$ be as in subsection 3.2.1. Then $\omega_{X / S}$ is big and if $\phi: X \rightarrow Z$ is the birational map induced by $\omega_{X / S}^{m}$, then $\phi$ separates the fibers of $f$ over an open dense subset of $S$ and $\left.\phi\right|_{X_{g e n}}$ is equal to the map induced by $\omega_{X_{g e n}}^{m}$ for $m$ large and divisible enough.

Proof. Let $l>0$ be divisible enough and such that $\omega_{X_{\text {gen }}}^{l}$ is generated by global sections. Then by [Viehweg83b, Thm. II] there exists an ample locally free sheaf $\mathcal{A}$ on $S$ and an injective morphism $\alpha: \mathcal{A} \rightarrow \operatorname{Sym}^{r}\left(f_{*} \omega_{X / S}^{l}\right)$ for some $r>0$, such that $\alpha$ is an isomorphism over a dense open subvariety, $U \subseteq S$. The statement follows by applying Lemma 3.1 .3 with $\mathcal{M}=\omega_{X_{\text {gen }}}^{l}$.

3.2.4 Definition (Esnault-Viehweg90). Let $F$ be a normal Gorenstein variety with rational singularities, $\mathcal{L}$ a line bundle on $F$ and $\Gamma$ an effective divisor such that $\mathcal{L}=\mathcal{O}_{F}(\Gamma)$. Let $\mathcal{C}(\Gamma, N)=\operatorname{coker}\left(\tau_{*} \omega_{\tilde{F}}\left(-\left[\frac{\tilde{\Gamma}}{N}\right]\right) \rightarrow \omega_{F}\right)$, where $\tau: \tilde{F} \rightarrow F$ is a resolution of singularities such that $\tilde{\Gamma}=\tau^{*} \Gamma$ is a normal crossing divisor, $e(\Gamma)=$ $\min \left\{N \in \mathbb{N}_{+} \mid \mathcal{C}(\Gamma, N)=0\right\}$, and $e(\mathcal{L})=\sup \left\{e(\Gamma) \mid \exists \lambda \in H^{0}(F, \mathcal{L})\right.$ such that $\Gamma=$ $(\lambda=0)\}$. e $(\mathcal{L})$ will be called the Esnault-Viehweg threshold of $\mathcal{L}$. For properties of $e(\mathcal{L})$ the reader should consult [Viehweg95, §5.3-4].

Using the notation of subsection 2.1, $r(m)$ will denote the rank of $f_{*} \omega_{X / S}^{m}$. This is equal to the $m$ th plurigenus of the general fiber of $f, P_{m}\left(X_{\text {gen }}\right) . e(m)$ will denote the Esnault-Viehweg threshold of $\omega_{X_{\text {gen }}}^{m}$. If $\omega_{X_{\text {gen }}}$ is ample, then $e(m) \leq m^{n} K_{X_{\text {gen }}}^{n}+1$ for $m \gg 0$ by [Viehweg95, 5.12].

The following statement gives an effective measure of the positivity of $f_{*} \omega_{X / S}^{m}$. The main ideas of the proof originate in the works of Kawamata, Kollár and Viehweg. However, there are some essential differences compared to the many 
variations that have appeared in the literature so far. Since the base is higher dimensional the statement is somewhat weaker than in the case when the base is a curve. It turns out that for the applications here this statement is sufficient. Another difference is a trivial, but crucial improvement: It is not necessary to start the proof by passing to a finite cover as has been customary.

3.2.5 Proposition. Let $f: X \rightarrow S$ be as in subsection 3.2.1, and $m$ sufficiently large and divisible. Then there exists a sheaf $\mathcal{F}$ weakly positive over $S \backslash \Delta$ and an embedding

$$
\iota: \mathcal{F} \hookrightarrow \operatorname{Sym}^{e(m) r(m)}\left(f_{*} \omega_{X / S}^{m}\right) \otimes\left(\operatorname{det} f_{*} \omega_{X / S}^{m}\right)^{-1}
$$

such that $\iota$ is an isomorphism on $S \backslash \Delta$.

Proof. Let $\mathcal{D}=\operatorname{det} f_{*} \omega_{X / S}^{m}, q=e(m) r(m), \pi: Z \rightarrow X^{q}=X \times_{S} X \times_{S} \cdots \times_{S}$ $X$ be a resolution of singularities, $\rho=f^{q} \circ \pi: Z \rightarrow S$, and $\mathcal{M}=\pi^{*} \omega_{X^{q} / S}=$ $\pi^{*}\left(\otimes \operatorname{pr}_{i}^{*} \omega_{X / S}\right)$. By the assumptions $\rho_{*} \mathcal{M}^{m}$ is locally free on $S$, hence $\mathcal{M}$ is big by Lemma 3.2.3. $f^{q}$ is a Gorenstein morphism and the fibers over $S \backslash \Delta$ have rational singularities, so there are natural injective maps:

$$
\begin{aligned}
\mathcal{D}^{e(m)} & \hookrightarrow\left(f_{*} \omega_{X / S}^{m}\right)^{\otimes q} \simeq f_{*}^{q} \omega_{X^{q} / S}^{m}, \\
f_{*}^{q} \omega_{X^{q} / S}^{m} & \hookrightarrow \rho_{*} \mathcal{M}^{m}, \\
\left(\rho_{*}\left(\mathcal{M}^{m-1} \otimes \omega_{Z / S}\right)\right)^{* *} & \hookrightarrow f_{*}^{q} \omega_{X^{q} / S}^{m}
\end{aligned}
$$

where (3.2.5.2) and (3.2.5.3) are isomorphisms over $S \backslash \Delta$. The composition of 3.2.5.1) and 3.2.5.2) gives an injective map $\rho^{*} \mathcal{D}^{e(m)} \rightarrow \rho^{*} \rho_{*} \mathcal{M}^{m} \rightarrow \mathcal{M}^{m}$ and hence a section $\sigma \in H^{0}\left(Z, \mathcal{M}^{m} \otimes \rho^{*} \mathcal{D}^{-e(m)}\right)$. Let $A=(\sigma=0)$. Since $\pi$ was an arbitrary resolution of singularities, one may replace it by further blow-ups. In particular, one may assume that $A$ is a normal crossing divisor.

Let $\mathcal{J} \subseteq \mathcal{O}_{Z}$ be the ideal sheaf defined as $\operatorname{im}\left[\rho^{*} f_{*}^{q} \omega_{X^{q} / S}^{m} \rightarrow \mathcal{M}^{m}\right]=\mathcal{M}^{m} \otimes \mathcal{J}$. Note that $\operatorname{supp} \mathcal{O}_{X} / \mathcal{J} \subseteq \operatorname{supp} f^{-1} \Delta$. By blowing up $\mathcal{J}$ one can assume that it is a line bundle, trivial over $X \backslash f^{-1} \Delta$. By Viehweg89. II, 2.7(ii)] $f_{*} \omega_{X / S}^{m}$ is weakly positive over $S \backslash \Delta$, so $\mathcal{M}^{m} \otimes \mathcal{J}$ is weakly positive over $X \backslash f^{-1} \Delta$. Since $\mathcal{J}$ is trivial over $X \backslash f^{-1} \Delta$, one obtains that $\mathcal{M}$ itself is weakly positive over $X \backslash f^{-1} \Delta$.

Let $\mathcal{L}$ be an ample line bundle on $X$ and $\mathcal{K}=\mathcal{M}^{m-1} \otimes \rho^{*} \mathcal{D}^{-1}$. Since $\mathcal{N}=$ $\mathcal{K}^{e(m)}(-A)=\mathcal{M}^{e(m)(m-1)-m}$ is big, there exists an $a>0$ such that $\mathcal{N}^{a} \otimes \mathcal{L}^{-1} \simeq$ $\mathcal{O}_{X}(B)$ for an effective divisor $B$ and by further blowing up $Z$ we may assume that $A+B$ is a normal crossing divisor. Since $\mathcal{N}$ is weakly positive over $X \backslash f^{-1} \Delta, \mathcal{N}^{N}(-B)=\mathcal{N}^{N-a} \otimes \mathcal{L}$ is semi-ample with respect to $X \backslash f^{-1} \Delta$ for all $N>a$. For $N \gg 0,\left[\frac{N \cdot A+B}{N \cdot e(m)}\right]=\left[\frac{A}{e(m)}\right]$, so by Viehweg89, II, 4.3 and III, 2.6] $\rho_{*}\left(\mathcal{K} \otimes \omega_{Z / S}(-[A / q e(m)])\right)$ is weakly positive over $S \backslash \Delta$ and by Viehweg95, 5.14, 5.21], $\rho_{*}\left(\mathcal{K} \otimes \omega_{Z / S}(-[A / e(m)])\right) \hookrightarrow \rho_{*}\left(\mathcal{K} \otimes \omega_{Z / S}\right)$ is an isomorphism on $S \backslash \Delta$. Then using the fact that $\left(\rho_{*}\left(\mathcal{K} \otimes \omega_{Z / S}\right)\right)^{* *}$ is isomorphic to $\left(\rho_{*}\left(\mathcal{M}^{m-1} \otimes \omega_{Z / S}\right)\right)^{* *} \otimes \mathcal{D}^{-1}$, one concludes that $\rho_{*}\left(\mathcal{K} \otimes \omega_{Z / S}(-[A / e(m)])\right)$ is a subsheaf of $\left(f_{*} \omega_{X / S}^{m}\right)^{\otimes q} \otimes \mathcal{D}^{-1}$ such that they are isomorphic on $S \backslash \Delta$ by (3.2.5.3). Let $\eta:\left(f_{*} \omega_{X / S}^{m}\right)^{\otimes q} \otimes \mathcal{D}^{-1} \rightarrow \operatorname{Sym}^{q}\left(f_{*} \omega_{X / S}^{m}\right) \otimes \mathcal{D}^{-1}$ be the natural map. Then the sheaf $\mathcal{F}=\eta\left(\rho_{*}\left(\mathcal{K} \otimes \omega_{Z / S}(-[A / e(m)])\right)\right)$ has all the desired properties.

3.2.6 Corollary. Using the notation and assumptions of Proposition 3.2.5, let $\mathcal{N}$ be a line bundle on $S$ such that $\operatorname{det} f_{*} \omega_{X / S}^{m} \otimes \mathcal{N}^{m e(m) r(m)}$ is ample. Assume that 
$\omega_{X_{t}}$ is big and 1-ample for $t \in S \backslash \Delta$. Then $\omega_{X / S} \otimes f^{*} \mathcal{N}$ is big and 1-ample with respect to $X \backslash f^{-1} \Delta$.

Proof. By Proposition 3.2.5. Sym ${ }^{e(m) r(m)}\left(f_{*} \omega_{X / S}^{m} \otimes \mathcal{N}^{m}\right)$ contains a weakly positive sheaf that is equal to it on $S \backslash \Delta$. Then by Lemma 3.1.3 $\omega_{X / S}^{m} \otimes f^{*} \mathcal{N}^{m}$ is big and 1-ample with respect to $X \backslash f^{-1} \Delta$.

\section{Boundedness}

4.1 Theorem. Let $f: X \rightarrow S$ be a smooth morphism of smooth projective varieties. Assume that $\operatorname{Var}(f)=\operatorname{dim} S$ and $X_{\text {gen }}$ is either a minimal surface of general type or a canonically polarized variety. Then for every ample line bundle $\mathcal{K}$ on $S$ there exists a constant $\nu=\nu(n, S, \mathcal{K})$ depending only on $n, S$, and $\mathcal{K}$ such that $\operatorname{det} f_{*} \omega_{X / S}^{m} \otimes$ $\mathcal{L}^{-m e(m) r(m)}$ is not ample for any line bundle $\mathcal{L}$ that contains $\mathcal{K}^{\nu}$. In particular, $\operatorname{det} f_{*} \omega_{X / S}^{m} \otimes \mathcal{K}^{-\nu m e(m) r(m)}$ is not ample.

Proof. Let $n=\operatorname{dim} X$. For a line bundle $\mathcal{L}$ on $S$ such that $\operatorname{det} f_{*} \omega_{X / S}^{m} \otimes \mathcal{L}^{-m e}(m) r(m)$ is ample, $\omega_{X / S} \otimes f^{*} \mathcal{L}^{-1}$ is big and 1-ample by Corollary 3.2.6. Let $\mathcal{K}$ be an ample line bundle on $S$ and let $\nu=\nu(n, S, \mathcal{K})$ be such that $\mathcal{K}^{\nu} \otimes \mathcal{E}$ is ample for all $\mathcal{E} \in \mathfrak{T}^{i}\left(\mathcal{O}_{S}\right), i=0, \ldots, n-k$. Finally let $\mathcal{L}_{\nu}=\omega_{X / S} \otimes f^{*}\left(\mathcal{L}^{-1} \otimes \mathcal{K}^{\nu}\right)$. Then $\mathcal{L}_{\nu} \otimes f^{*} \mathcal{E}$ is big and 1-ample for all $\mathcal{E} \in \mathfrak{T}^{i}\left(\mathcal{O}_{S}\right), i=0, \ldots, n-k$. Now if $\mathcal{K}^{\nu} \subseteq \mathcal{L}$, then $\mathcal{L}_{\nu} \subseteq \omega_{X / S}$ so $H^{n}\left(X, \omega_{X}\right)=H^{n}\left(X, f^{*} \omega_{S} \otimes \mathcal{M}\right)=0$ follows by Corollary 2.8 Since $H^{n}\left(X, \omega_{X}\right) \neq 0$, this finishes the proof.

4.2 Corollary. If in addition $\rho(S)=1$, then there exists a constant $\nu(n, S)$ such that

$$
\operatorname{deg} f_{*} \omega_{X / S}^{m} \leq \nu(n, S) m e(m) r(m) .
$$

\section{HYPERBOLICITY}

The vanishing theorems obtained in $\S 2$ put restrictive constraints on the positivity of $\omega_{X}$ if $\omega_{S}(\Delta)^{-1}$ is nef. In particular, if $S$ has non-positive Kodaira dimension, then one expects that there will be no semi-stable families with small $\Delta$. A more precise formulation of this follows.

5.1. $S$ is an abelian variety. This case was already studied in Kovács97a. A slight generalization of the results of that article is presented here.

5.1.1 Theorem. Let $A$ be an abelian variety and $f: X \rightarrow A$ a smooth morphism. Assume that for all $t \in A, X_{t}$ is either a minimal surface of general type or a canonically polarized variety. Then $\operatorname{Var}(f)<\operatorname{dim} A$.

Proof. Suppose that $\operatorname{Var}(f)=\operatorname{dim} A$. Then $\omega_{X}$ is big and 1-ample by Lemma 3.2 .3 Since $A$ is an abelian variety, there exists a choice of filtrations on $A$, such that $\mathfrak{T}^{i}\left(\mathcal{O}_{A}\right)=\left\{\mathcal{O}_{A}\right\}$. Then by Corollary $2.9, H^{n}\left(X, \omega_{X}\right)=0$, a contradiction.

From this, using the technique of [Kovács97a, §2], one can easily derive the following:

5.1.2 Corollary. Let $A$ be an abelian variety and $f: X \rightarrow A$ a smooth morphism. Assume that for all $t \in A, X_{t}$ is either a minimal surface of general type or a canonically polarized variety. Then $f$ is isotrivial. 


\section{2. $S$ is a projective space or a quadric.}

5.2.1 Conjecture. (i) Let $f: X \rightarrow \mathbb{P}^{k}$ be a family of varieties of general type and $\Delta \subset \mathbb{P}^{k}$ an effective divisor such that $X \backslash f^{*} \Delta \rightarrow \mathbb{P}^{k} \backslash \Delta$ is smooth. Assume that $\operatorname{Var}(f)=k$. Then $\operatorname{deg} \Delta>k+1$. Or more generally,

(ii) Let $f: X \rightarrow S$ be a family of varieties of general type and $\Delta \subset S$ an effective divisor such that $X \backslash f^{*} \Delta=\rightarrow S \backslash \Delta$ is smooth. Assume that $\operatorname{Var}(f)=\operatorname{dim} S$ (and perhaps that $\rho(S)=1$ if needed). Then $\omega_{S}(\Delta)$ is big.

Conjecture 5.2.1(i) holds for $k=1$ by [Beauville81, Migliorini95, [Kovács96, Kovács97b], Bedulev-Viehweg00, [Kovács00a], Kovács00b], |Viehweg-Zuo01a]. Here we prove this conjecture for semi-stable families of curves and $k \leq 3$; cf. Viehweg00, 2.6].

5.2.2 Theorem. Let $f: X \rightarrow \mathbb{P}^{k}$ be a semi-stable family of curves of genus at least 2 and $\Delta \subset \mathbb{P}^{k}$ a normal crossing divisor such that $X \backslash f^{*} \Delta \rightarrow \mathbb{P}^{k} \backslash \Delta$ is smooth. Assume that $k \leq 3$ and that there exists an effective divisor $E$ on $X$ such that $\omega_{X}(-E)$ is ample. Then $\operatorname{deg} \Delta>k+1$.

Proof. Suppose that $\operatorname{deg} \Delta \leq k+1$. Then $\omega_{\mathbb{P}^{k}}(\Delta)^{-1}$ is nef, so $\mathcal{M}=f^{*} \omega_{\mathbb{P}^{k}}(\Delta)^{-1} \otimes$ $\omega_{X}$ contains an ample line bundle, $\mathcal{L}=f^{*} \omega_{\mathbb{P}^{k}}(\Delta)^{-1} \otimes \omega_{X}(-E)$. It is easy to see that $\Omega_{\mathbb{P}^{k}}^{q}(\log \Delta) \otimes \omega_{\mathbb{P}^{k}}^{-1}$ is semipositive for $q=0, \ldots, k$. Since $k \leq 3$, all of these sheaves have rank at most $k$, so if one chooses the trivial filtration for all locally free sheaves on $S$, then $\mathcal{L}\left(f^{*} \Delta\right) \otimes f^{*} \mathcal{E}$ is ample for all $\mathcal{E} \in \mathfrak{T}^{i}\left(\mathcal{O}_{S}\right), i=0,1$. Then by Corollary [2.8, $H^{n}\left(X, \omega_{X}\right)=0$, a contradiction.

5.2.3 Remark. The reason for the restriction $k \leq 3$ is that this way $\Omega_{\mathbb{P} k}^{q}(\log \Delta) \otimes \omega_{\mathbb{P} k}^{-1}$ has at most rank $k$ for any $q$. If one finds a filtration of these sheaves such that the associated quotients are semi-positive, then this assumption can be removed. The restriction to families of curves is for the same reason. To allow higher-dimensional fibers one has to find suitable filtrations of tensor products of the sheaves of logarithmic forms.

An argument, similar to the above, proves Conjecture 5.2.1(ii) for a smooth quadric hypersurface of dimension at most three.

5.2.4 Theorem. Let $Q \subset \mathbb{P}^{k+1}$ be a smooth quadric hypersurface, $f: X \rightarrow Q$ a semi-stable family of curves of genus at least 2 and $\Delta \subset Q$ a normal crossing divisor such that $X \backslash f^{*} \Delta \rightarrow Q \backslash \Delta$ is smooth. Assume that $k \leq 3$ and that there exists an effective divisor $E$ supported on $\operatorname{supp} f^{*} \Delta$ such that $\omega_{X}(-E)$ is ample. Then $\omega_{Q}(\Delta)$ is ample, i.e., $\operatorname{deg}_{\mathbb{P}^{k+1}} \Delta>2 k$.

Proof. If $\omega_{Q}(\Delta)^{-1}$ is nef or has a non-zero global section, then $\mathcal{M}=f^{*} \omega_{Q}(\Delta)^{-1}$ $\otimes \omega_{X}$ contains an ample line bundle, $\mathcal{L}=f^{*} \omega_{Q}(\Delta)^{-1} \otimes \omega_{X}(-E)$. The rest of the proof works the same way as above.

\section{ACKNOWLEDGEMENTS}

This article was finished while I enjoyed the hospitality of the Isaac Newton Institute for Mathematical Sciences as a participant of the Higher Dimensional Geometry Programme. The organizers, participants, and staff helped make my stay there a productive one. I would like to thank the referee for saving me from committing an embarrassing error and for many useful suggestions. 


\section{REFERENCES}

[Abramovich-Karu00] D. Abramovich, K. Karu, Weak semistable reduction in characteristic 0, Invent. Math. 139 (2000) 241-273. MR 2001f:14021

[Angehrn-Siu95] U. Angehrn, Y.T. Siu, Effective freeness and point separation for adjoint bundles, Invent. Math. 122 (1995) 291-308. MR 97b:32036

[Arakelov71] S. Arakelov, Families of algebraic curves with fixed degeneracies, Izv. A. N. SSSR 35 (1971) 1269-1293. MR 48:298

[Beauville81] A. Beauville, Le nombre minimum de fibres singulières d'une courbe stable sur $\mathbb{P}^{1}$, Astérisque 86 (1981) 97-108.

[Bedulev-Viehweg00] E. Bedulev, E. Viehweg, On the Shafarevich conjecture for surfaces of general type over function fields, Inv. Math. 139 (2000) 603-615. MR 2001f: 14065

[Esnault-Viehweg90] H. Esnault, E. Viehweg, Effective bounds for semipositive sheaves and for the height of points on curves over complex function fields, Compositio Math. 76 (1990) 69-85. MR 91m:14038

[Esnault-Viehweg92] H. Esnault, E. Viehweg, Lectures on vanishing theorems, DMV Seminar 20, Birkhäuser (1992). MR 94a:14017

[Faltings83] G. Faltings, Arakelov's Theorem for abelian varieties, Inv. Math. 73 (1983) 337-348. MR 85m:14061

[Karu00] K. Karu, Minimal models and boundedness of stable varieties, J. Alg. Geom. 9 (2000) 93-109. MR 2001g:14059

[Kollár87] J. Kollár, Subadditivity of the Kodaira dimension: Fibers of general type, Advanced Studies in Pure Math. 10, Algebraic Geometry Sendai 85, Kinokuniya-North Holland (1987) 361-398. MR 89i:14029

[Kollár90] J. Kollár, Projectivity of Complete Moduli, J. Diff. Geom. 32 (1990) 235268. MR 92e:14008

[Kovács96] S. J. Kovács, Smooth families over rational and elliptic curves, J. Alg. Geom. 5 (1996) 369-385. MR 97c:14035

[Kovács97a] S. J. Kovács, Families over a base with a birationally nef tangent bundle, Math. Ann. 308 (1997) 347-359. MR 98h:14039

[Kovács97b] S. J. Kovács, On the minimal number of singular fibres in a family of surfaces of general type, J. reine angew. Math. 487 (1997) 171-177. MR 98h:14038

[Kovács00a] S. J. Kovács, Algebraic hyperbolicity of fine moduli spaces, J. Alg. Geom. 9 (2000) 165-174. MR 2000i:14017

[Kovács00b] S. J. Kovács, Logarithmic vanishing theorems and Arakelov-Parshin boundedness for singular varieties, Comp. Math. 131 (2002) 291-317. MR 2003a:14025

[LePotier75] J. Le Potier, Annulation de la cohomologie à valeurs dans un fibré vectoriel holomorphe positif de rang quelconque, Math. Ann. 218 (1975) 35-53. MR 52:6044

[Migliorini95] L. Migliorini, A smooth family of minimal surfaces of general type over a curve of genus at most one is trivial, J. Alg. Geom. 4 (1995) 353-361. MR 95m:14023

[Parshin68] A. Parshin, Algebraic curves over function fields, Izv. A. N. SSSR 32 (1968) 1145-1170. MR 41:1740

[Shiffman-Sommese85] B. Shiffman, A. J. Sommese, Vanishing theorems on complex manifolds, Progress. in Math. 56, Birkhäuser (1985). MR 86h:32048

[Viehweg83a] E. Viehweg, Weak positivity and the additivity of Kodaira dimension for certain fibre spaces, Advanced Studies in Pure Math. 1, Algebraic Varieties and Analytic Varieties (1983) 329-353. MR 85b:14041

[Viehweg83b] E. Viehweg, Weak positivity and the additivity of the Kodaira dimension II, Classification of algebraic and analytic manifolds, Birkhäuser (1983) 567-590. MR 85i:14020

[Viehweg89] E. Viehweg, Weak positivity and the stability of certain Hilbert points, Inv. Math. 96 (1989) 639-667; II 101 (1990) 191-223; III 101 (1990) 521-544. MR 90i:14037. MR 91f:14032, MR 91f:14032 
[Viehweg95]

E. Viehweg, Quasi-Projective Moduli of Polarized Manifolds, Springer (1995). MR 97j:14001

[Viehweg00]

E. Viehweg, Positivity of direct image sheaves and applications to families of higher dimensional manifolds, Lecture notes, "School on Vanishing Theorems and Effective Results in Algebraic Geometry", ICTP, Trieste, (2000).

[Viehweg-Zuo01a] E. Viehweg, K. Zuo, On the isotriviality of families of projective manifolds over curves, J. Alg. Geom. 10 (2001) 781-799. MR 2002g:14012

[Viehweg-Zuo01b] E. Viehweg, K. Zuo, Base spaces of non-isotrivial families of smooth minimal models, preprint (2001).

[Viehweg-Zuo01c] E. Viehweg, K. Zuo, On the Brody hyperbolicity of moduli spaces for canonically polarized manifolds, preprint (2001).

Department of Mathematics, University of Washington, 354350, Seattle, Washington 98195

E-mail address: kovacs@math.washington.edu

URL: http://www. math. washington. edu/ ${ }^{*}$ kovacs 